Gazi University
Journal of Science
http://dergipark.gov.tr/gujs

\title{
ARM MCU-Based Experimental EEG Signal Generator Using Internal DAC and PWM Outputs
}

\author{
Ertugrul KARAKULAK* (iD \\ Tekirdag Namik Kemal University, Vocational School of Technical Sciences, Biomedical Device Technology Department, Tekirdag, Turkey
}

\author{
Highlights \\ - This paper focuses on a bio-potential signal generator. \\ - Signal generator using ARM-based Microcontroller. \\ - Signal generation via DAC and PWM outputs.
}

\section{Article Info \\ Received: 14 Jan 2021 \\ Accepted: 05 Aug 2021

\begin{tabular}{l} 
Keywords \\
\hline Signal generator \\
Electroencephalograpram \\
Microcontroller \\
Pulse width modulation
\end{tabular}

\section{INTRODUCTION}

Bio-potential signals, such as ECG (Electrocardiography), EEG (Electroencephalography) and so on are widely used in biomedical education, scientific research and testing of medical devices. For example, signal generators are applied during the calibration of devices used in health institutions. Such generators cannot be purchased in sufficient numbers, especially for educational purposes, due to their cost. Since microcontroller-based signal generators can be made with easily accessible and cheap materials, they are included in experiment sets especially in education laboratories and are quite common [1].

EEG signals are electrophysiological signals which are emitted from the brain. These signals are used in many applications and researches, from the detection of anomalies to the control of prostheses $[2,3]$. There are studies already done on EEG signal generators in the literature. Shakir et al. proposed a generator that can generate EEG signals using model-based EEG signals and experimental EEG signals [4]. The hardware which they designed can produce EEG signals of normal, pre-seizure and seizure states. Tejkl at al. proposed an EEG signal generator made of a signal generator, frequency divider, and Opamps. The circuit which they proposed can produce random EEG signal [5]. In addition, in the marketplace, there are also EEG generators that produce EEG signals and can be used in device tests and research studies. These devices generate non-experimental signals [6]. Many signal generators have been proposed in the literature to generate other electrophysiological signals. Yener et al. proposed an Arduino-based circuit for generating the experimental ECG signal obtained from databases. In the system which they designed, the ECG trace is generated via the PWM (Pulse Width Modulation) output of the microcontroller [7]. Zhang et al. proposed a Microchip PIC microcontroller-based ECG signal generator. The system, which includes a DAC

* e-mail: ekarakulak@nku.edu.tr 
(Digital Analog Converter), produces non-experimental, ECG signals belonging to an ECG signal model [8]. In [9], Wei et al. proposed a microcontroller-based system that produces ECG signals of 4 leads with McSharry's ECG model [10].

In this study, a signal generator that produces experimentally obtained EEG signals, which is needed by biomedical laboratories is proposed. This signal generator obtains experimental signals from an SD card. Two methods are suggested for the generation of the signal, PWM and DAC outputs of the Microcontrollers. It has been shown that the experimentally obtained EEG signals can be produced by both methods.

There are many free databases on the Internet where experimental bio-potential signals are available. The data used in this study are taken from the University of California's open database [11]. The EEG data taken are recorded on an SD card in text format. The microcontroller read the recorded data from the SD card and produce outputs corresponding to this data via the DAC and PWM ports.

The rest of the paper is organized as follows: in the second section, an acquired EEG data taken from a database is shown; in the third section, the EEG generator system is introduced and its circuit scheme is given. The fourth section provides the experimental results of the proposed circuit; the paper is concluded with a fifth section.

\section{AN EXPERIMENTAL EEG WAVEFORM}

There are numerous databases on the internet which provide free open-access experimental bio-potential signals. In this study, experimental EEG data are obtained from the open-access database of the University of California [11]. The open access database provides the experimental EEG signals acquired from different lobes. Any of the recorded signals can be used to obtain the voltage output of the signal generator designed in this study. In this study, the data of probe number 1 on the frontal lobe (FP1) are preferred to show the applicability of the concept. In addition, there are EEG signals regarding various anomalies and various stimulation results (Evoked Potentials) in databases. The experimental data drawn with Matlab ${ }^{\mathrm{TM}}$ can be seen in Figure 1. The length of the data used for this study is 255 data points at $4.3 \mathrm{~ms}$ intervals.

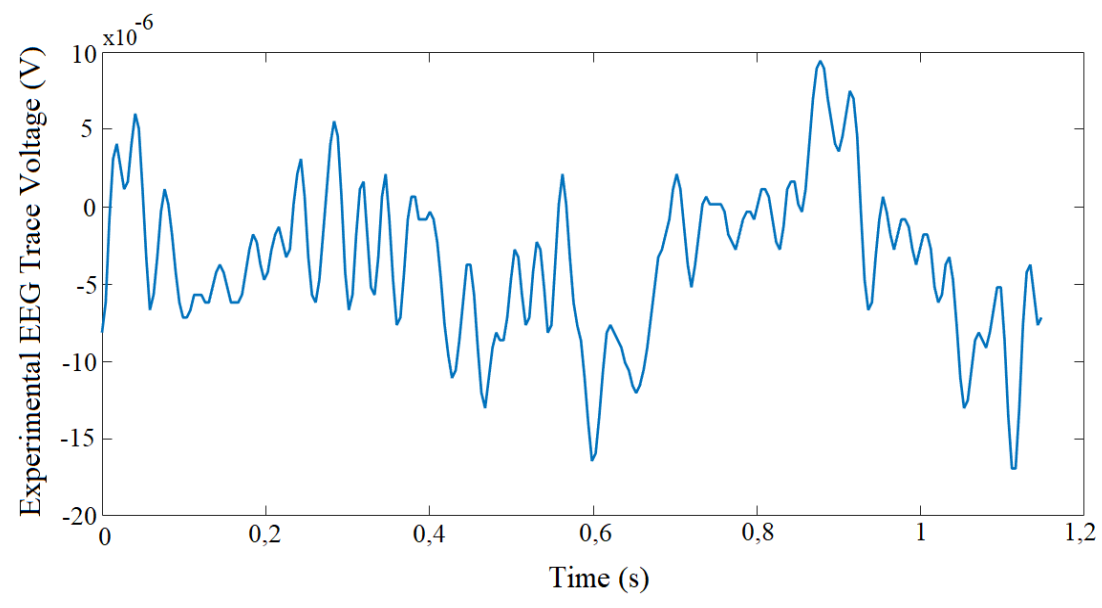

Figure 1. The experimental EEG trace is taken from the open-access database [11] 


\section{SIGNAL GENERATOR CIRCUIT}

The proposed circuit is implemented on an STM32F429 Discovery development board where STM32F429 is an ARM architecture Microcontroller. The circuit diagram of the designed experimental EEG signal generator can be seen in Figure 2.

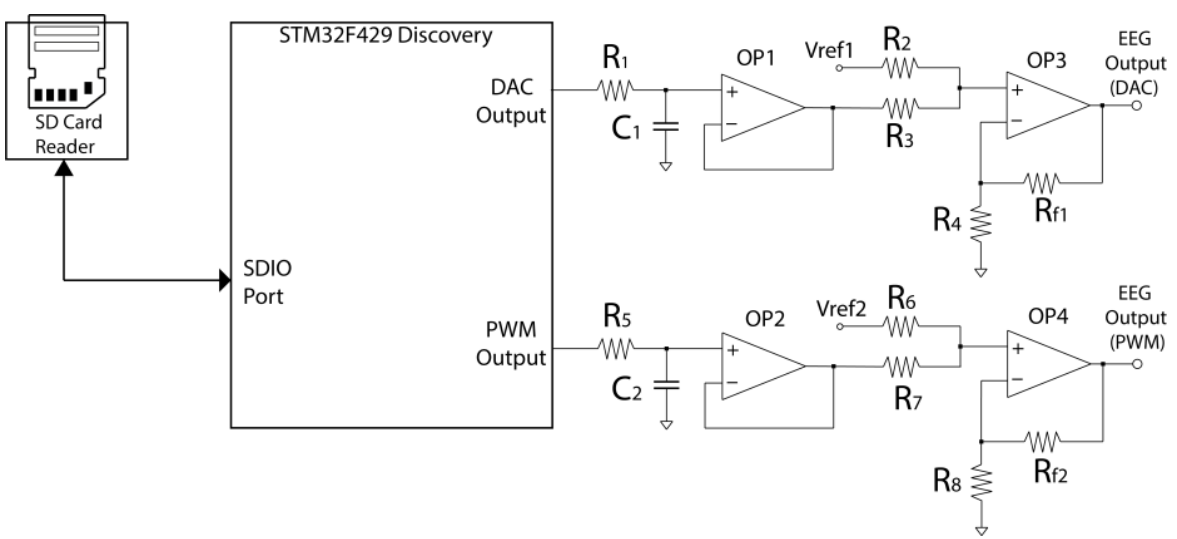

Figure 2. The Microcontroller Based EEG Signal Generator Circuit

Experimental data are recorded in text format on the SD card. SD card readers are included in such applications with their low prices, easy availability, and widespread use. SPI (Serial Peripheral Interface) or SDIO (Secure Digital Input Output) interfaces are mostly used for communication with SD cards. In this study, SDIO is preferred due to its easy configuration and faster communication ability. The EEG curve of the data taken from the SD card using the SDIO communication protocol can be seen in Figure 3. This curve has been obtained using the STM32CubeMonitor program and shows directly the data sequence which is received by the microcontroller from the SD Card. The quantization effects on the EEG signal can be seen in Figure 3 since the data series can be expressed using step functions of time, $t$. Equation (1) explains the quantization effects. For the time interval, $T_{s} \leq t \leq(\mathrm{n}+1) T_{s}$, the EEG signal in continuous time is given as

$x(t)=x[n] .\left(u\left(t-n T_{s}\right)-u\left(t-(n+1) T_{s}\right)\right)$

where $x[n]$ is the $n^{\text {th }}$ discrete value of on the EEG signal which is taken from SD Card, $u(t)$ is the unit step function and $T_{s}$ is the sampling period of the data sequence.

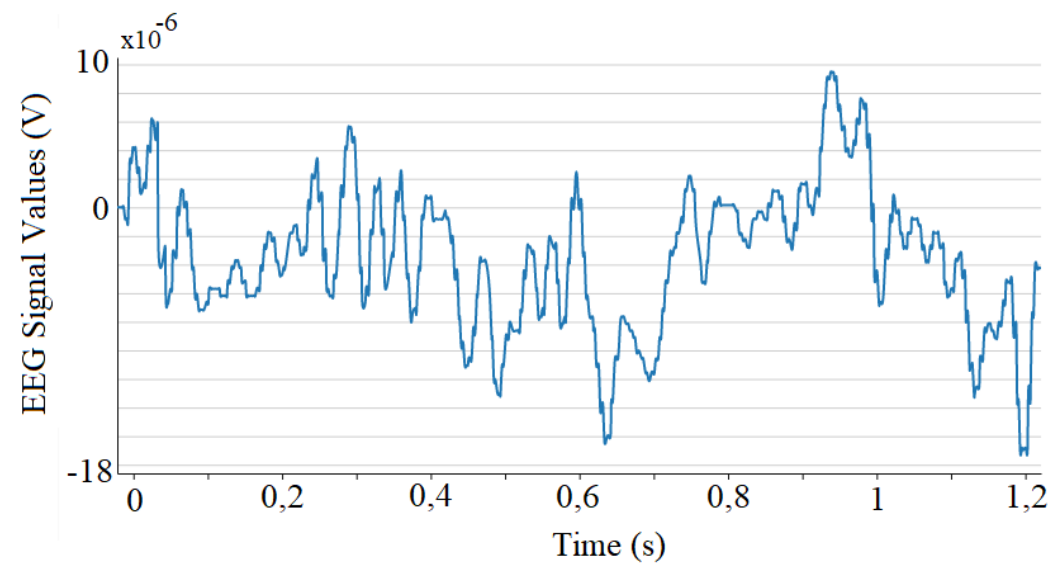

Figure 3. The EEG Trace is taken from SD Card Reader By Microcontroller 
One DAC port and one PWM port of the microcontroller are used separately to convert the data received from the SD card into analog signals. A passive Low Pass RC filter is placed at the PWM output and used to eliminate the quantization effects on the signal at the DAC output and to convert the PWM signal into DC. Voltage follower circuits with Opamps (OP1, OP2) are placed after RC filters at both outputs for isolation purposes. The transfer function of the filters is given in Equation (2)

$T(s)=1 /(1+s R C)$.

As seen in Figures 1 and 3, EEG signal has also the negative voltage levels. DAC and PWM ports of the microcontroller cannot generate a signal with negative voltage levels. For this reason, a summer circuit with opamp (OP3, OP4) is placed on both outputs and the voltage level is shifted with a DC offset to obtain a realistic EEG signal as necessary. The desired gain values can also be obtained with these circuits. The experimental setup can be seen in Figure 4. The filter and the opamp-based circuits are built on a protoboard.

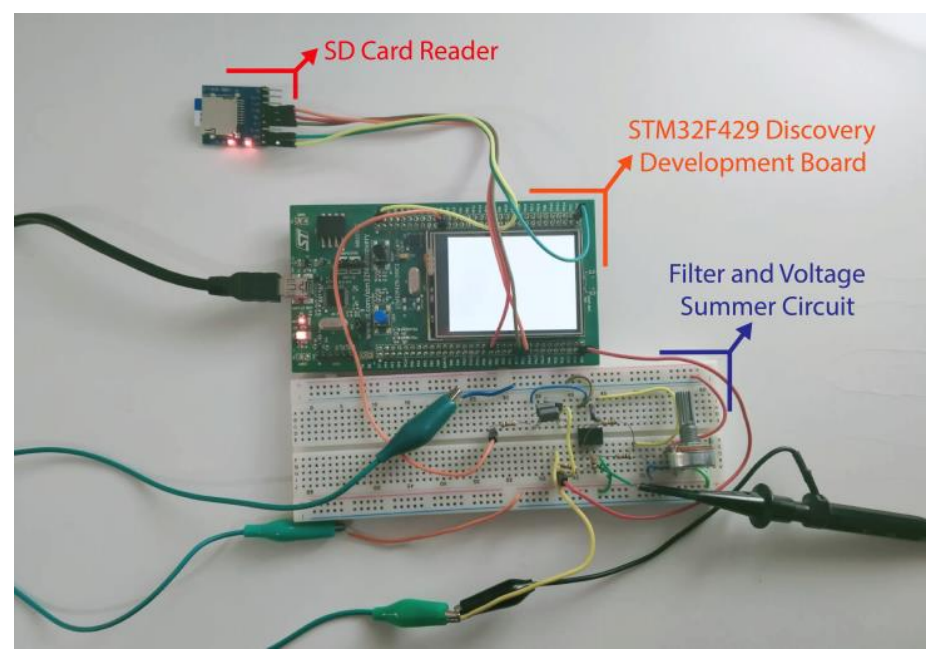

Figure 4. The experimental setup

\subsection{Signal Generation Via a DAC Output of Microcontroller}

First of all, an experimental EEG signal is generated using the internal DAC module of the microcontroller. A DAC module of the STM32F429 can work with 8 bit and 12 resolution configurations. In this study, the DAC module is configured for 12-bit resolution. The calculation of the value of the DAC port with the data received from the SD card can be seen in Equations (3)-(4). For the time interval of $n T_{s} \leq t \leq n T_{s} \mathrm{DAC}$ output voltage can be calculated in Equation (4)

$A[n]=\frac{2^{\text {res }}}{V_{\text {ref }}} \cdot x[n]$

$x(t)=\frac{V_{r e f}}{2^{\text {res }}} \sum_{k=0}^{k=r e s} A_{k}[n] .2^{k}$

where $T_{S}$ is the sampling period of the data, $x[n]$ is the $n^{\text {th }}$ discrete EEG value which is read from SD Card, $V_{r e f}$ is the reference voltage of the DAC port and res is the resolution of the DAC port of the microcontroller which is configured as 12 in our set up. $A[n]$ is the $n^{\text {th }}$ digital discrete value of the DAC module input, $x(t)$ is output voltage of the circuit, $A_{k}$ is $k^{\text {th }}$ digital value of DAC port. 
The filter at the DAC output is used to get rid of the quantization effects on the signal which is produced by the microcontroller. The frequency range of the EEG signals is known as $0.5 \mathrm{~Hz}-38 \mathrm{~Hz}$ [12]. The data set loaded on the SD card consists of samples taken at $4.3 \mathrm{~ms}$ intervals. In this case, the DAC value is renewed with approximately $250 \mathrm{~Hz}$. The values of the resistor and capacitor used in the low pass filter should be selected considering this sampling period.

\subsection{Signal Generation Via PWM Output of Microcontroller}

Almost all microcontrollers have built-in PWM ports nowadays. In this study, the PWM duty ratio value corresponding to the value taken from the SD card is recalculated and revised. For the time interval of $n T_{s} \leq t \leq n T_{s}$, the duty ratio of the PWM port used can be calculated in Equation (5)

$D(t)=\frac{X[n]}{V_{m}}$

where $V_{m}$ is the magnitude of the PWM output signal and $x[n]$ is the $n^{\text {th }}$ discrete EEG value which is taken from SD Card. The transfer function of the passive low-pass RC filter connected to the PWM port can be seen in Equation (1). The cut-off frequency of the filter should be determined according to the frequency range of the EEG signal $(0.5 \mathrm{~Hz}-38 \mathrm{~Hz})$ and the frequency of the generated PWM signal. In this study, the PWM signal frequency is set to $10 \mathrm{kHz}$.

\section{EXPERIMENTAL RESULTS}

In this section, the experimental results of the system are given.

\subsection{Experimental Results of Signal Generation Via DAC Output of Microcontroller}

The oscilloscope screenshots of the signals obtained from the DAC output of the circuit given in Figure 2 can be seen in Figure 5. Figure 5a shows the signal taken directly from the DAC output of the microcontroller. It should be noted that this signal voltage has only positive voltage values. A negative voltage cannot be taken from the microcontroller DAC output. Additionally, the quantization effects can be seen in the signal.

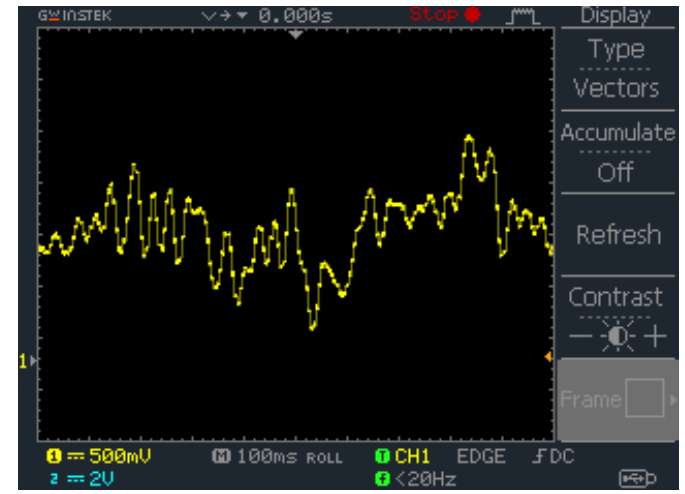

(a) 


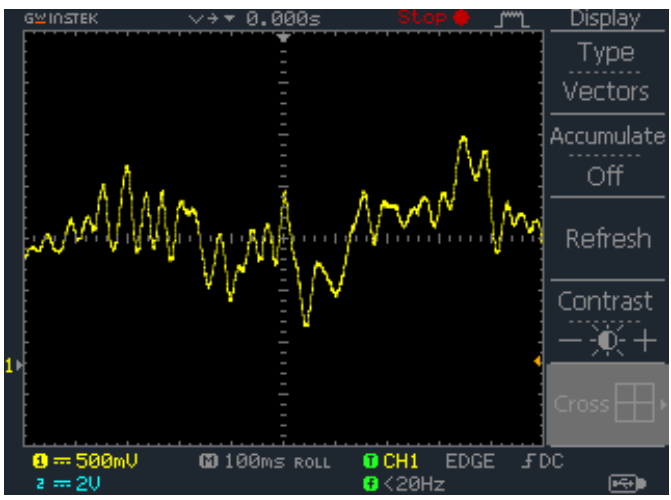

(b)

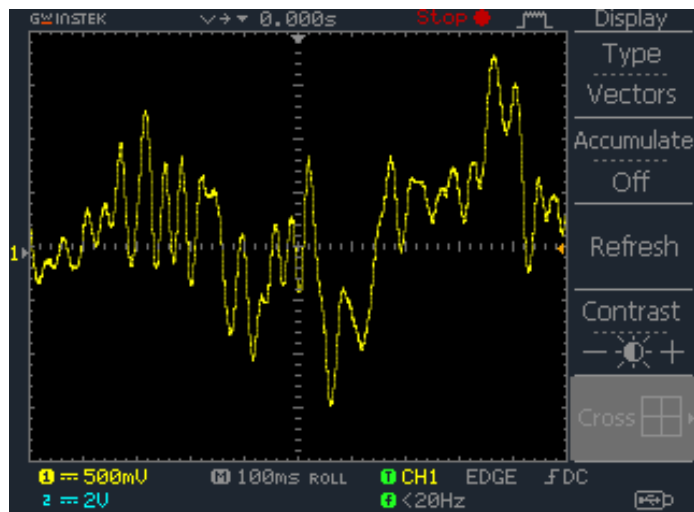

(c)

Figure 5. a) Output Voltage of The DAC Port, b) Output Voltage of The RC Filter of The DAC Port, and c) Output Voltage of The Summer Circuit of The DAC Port

Figure $5 \mathrm{~b}$ shows the signal at the output of the low pass RC filter of the DAC Port. It should be noted that the quantization is filtered on this waveform. The signal seen in Figure $5 \mathrm{c}$ is the output of the opamp-based summer circuit of the DAC Port. By applying the negative voltage $\mathrm{V}_{\text {ref1 }}$ to the one of the inputs of the summer circuit of the DAC Port, it is ensured that the EEG output signal takes both negative and positive voltage values as in reality.

\subsection{Experimental Results of Signal Generation Via PWM Output of Microcontroller}

The curves in Figure 6 are obtained from the output of the PWM port. It is seen that the EEG signal can be produced by the PWM Duty Ratio updated according to Equation (5) according to the data obtained from the SD card. Figure 6a shows the EEG signal taken from the output of the RC filter. It should be noted that the signal is in the positive voltage level. In Figure 6b, the PWM signal and the output of the RC filter are both given. A narrow range of the EEG signal is seen in Figure 6b. The change in the PWM duty cycle can be seen respectively the EEG signal. Figure $6 \mathrm{c}$ shows the signal at the output of the summer circuit. It can be seen that this signal has been shifted towards the negative voltage region. The system can run with also other EEG derivations, i.e. EEG signals acquired from different lobes since the ranges of the EEG signal magnitudes and frequencies are well-known. 


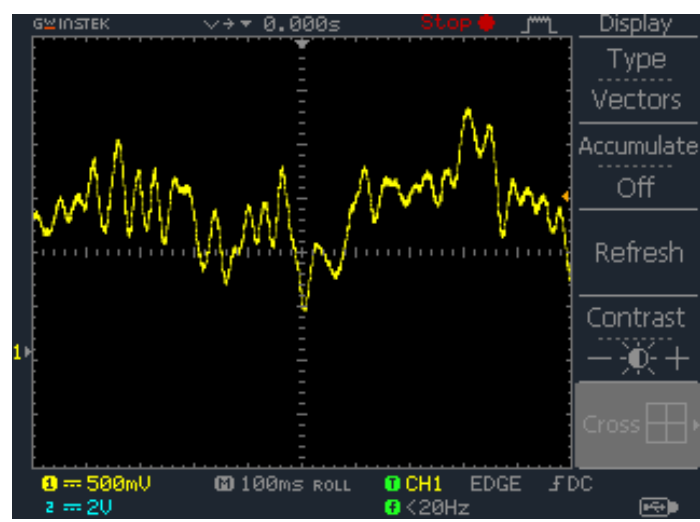

(a)

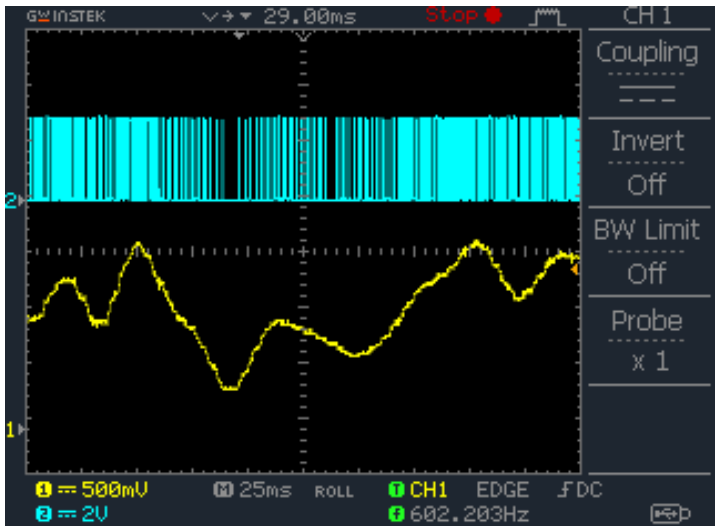

(b)

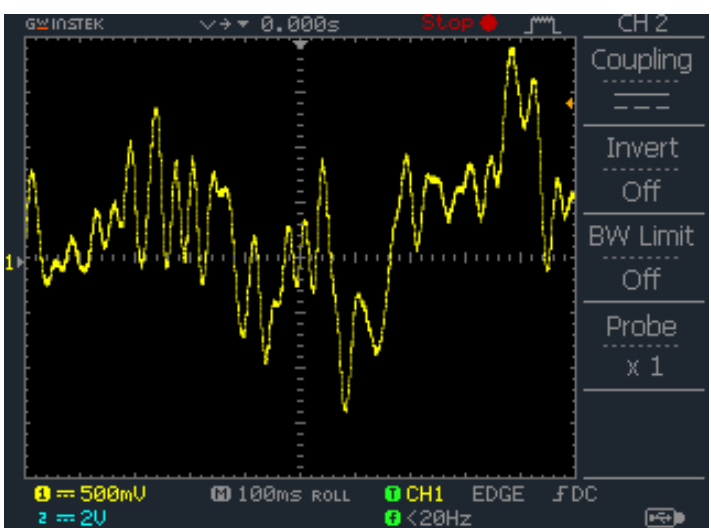

(c)

Figure 6. a) Output Voltage of The RC Filter of The PWM Port, b) PWM Output and The Filtered Voltage Signals, c) Output Voltage of The Summer Circuit of The PWM Port

\section{CONCLUSION AND DISCUSSION}

This study proposes a bio-potential signal generator that generates experimentally obtained EEG data using circuit components that can be found in every electronics laboratory. The signal generator generates the experimental data series which is read from an SD card. Two methods have been proposed to produce the experimental EEG signal and the results are given. One of them uses a DAC output port of the microcontroller and the other uses a PWM port of the microcontroller. It has been experimentally shown that the data from the database can be successfully produced with both methods. 
The data of probe number 1 on the frontal lobe (FP1) are used to make the signal generator in this study. However, any EEG data recorded and available can be used by the system. The system can be modified to have more output channels by using more PWM and/or DAC outputs. Also, the system can be modified to have a menu to choose from the EEG data list uploaded to the SD card. For example, the system can be modified to let the user choose from any EEG channel data (FP1, FP2, etc) within the database.

The proposed system produces experimentally obtained EEG data, unlike commercially available EEG signal generators. It can produce different types of EEG (Evoked potentials, seizures) from the database and different physiological signs (EOG, ERG, ECG, etc.) after some modifications. It can also generate multiple datasets taken from the database at the same time. Thus, multiple experimental channels of a record can be produced simultaneously. The system is made with cheap materials, that can be found easily, and then can be used in a laboratory.

STM32F429 Discovery development kit is used in the signal generator. Instead, it is possible to build the system with much cheaper options. For example, microcontrollers in the STM32F3 series and above have a DAC port and almost all microcontrollers in the marketplace have PWM ports. The researcher has chosen this development kit because of its availability. Moreover, with the addition of some hardware such as LCD, the system can serve as a permanent experiment set. In this way, it can become an important training tool in research laboratories and biomedical education laboratories.

The STM32F429 used in this study has only 2 internal DAC outputs. The most commonly used microcontrollers in the market do not have an internal DAC. Lack of or having a low number of DACs may not be sufficient for the simultaneous generation of many EEG channels. External DAC circuits may need to be installed to overcome this problem. The microcontroller used in this study can produce PWM independently from 56 channels at the same time with 14 Timers. Therefore, multiple EEG signals can be generated simultaneously using its PWM outputs.

\section{ACKNOWLEDGMENT}

The author would like to thank Dr. Şuayp Çağrı Yener for his help with software and Dr. Reşat Mutlu for proofreading.

\section{CONFLICTS OF INTEREST}

No conflict of interest was declared by the author.

\section{REFERENCES}

[1] Güçlü, B., "Low-cost computer-controlled current stimulator for the student laboratory", Advances in Physiology Education, 31(2): 223-231, (2007).

[2] Abiri, R., Borhani, S., Sellers, E. W., Jiang, Y., Zhao, X., “A comprehensive review of EEG-based brain-computer interface paradigms", Journal of Neural Engineering, 16(1), (2019).

[3] Balandong, R. P., Ahmad, R. F., Saad, M. N. M., Malik, A. S., "A review on EEG-based automatic sleepiness detection systems for driver”, IEEE Access, 6: 22908-22919, (2018).

[4] Shakir, M., Qidwai, U., Malik, A. S., Kamel, N., "Electroencephalography Simulation Hardware for Realistic Seizure, Preseizure and Normal Mode Signal Generation", Journal of Medical Imaging and Health Informatics, 5(1): 43-49, (2015). 
[5] Tejkl, L., Kudrna, P., Rafl, J., Svoboda, J., "Patient Simulators in Medical Education: New Enhancements", 2019 E-Health and Bioengineering Conference (EHB), Lasi, Romania, 1-4, (2019).

[6] https://www.netechcorp.us/Products/details/330-EEG-Simulator_71. Access date: 01.01.2021

[7] Yener, S. C., Mutlu, R., "A microcontroller-based ECG signal generator design utilizing microcontroller PWM output and experimental ECG data", 2018 Electric Electronics, Computer Science, Biomedical Engineerings' Meeting (EBBT), Istanbul, 1-4, (2018).

[8] Jun-an, Z., “The Design of ECG Signal Generator using PIC24F”, Procedia Engineering, 24: 523527, (2011).

[9] Wei, Y. C., Wei, Y. Y., Wey, S. T., Jang, L. S., "Design of a programmable ecg generator using a dynamical model", Applied Mechanics and Materials, 311: 485-490, (2013).

[10] McSharry, P. E., Clifford, G. D. , Tarassenko, L., Smith, L. A., "Dynamical model for generating synthetic electrocardiogram signals", IEEE Transactions On Biomedical Engineering, 50: 289294, (2003).

[11] https://sccn.ucsd.edu/ arno/fam2data/publicly_available_EEG_data.html. Access date: 01.01 .2021

[12] Campisi, P., La Rocca, D., "Brain waves for automatic biometric-based user recognition", IEEE Transactions on Information Forensics and Security, 9(5): 782-800, (2014). 